\title{
Renal Tubule
}

National Cancer Institute

\section{Source}

National Cancer Institute. Renal Tubule. NCI Thesaurus. Code C49274.

The anatomical segment of the kidney that is comprised of both the nephron and associated collecting tubules. These tubules or tubes contain fluid that has been filtered through the glomerulus. 